\title{
bases de un método macional para el dimensionamiento de refuerzos de firmes flexibles, basado en la medida de la deformación puntual de un eje cargado
}

SANDRO ROCCl, ingeniero de caminos

División de Materiales - Dirección General de Carreteras

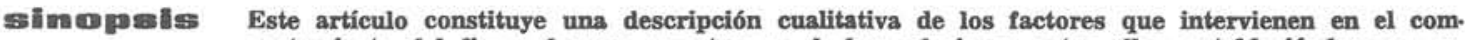
portamiento del firme de una carretera, y de las relaciones entre ellos, estableciéndose un esquema funcional cuyo objetivo último económico es la minimización del coste total (construc. cíón + conservación + explotación) de una carretera. Al mismo tiempo, se establecen las bases para un método de dimensionamiento de refuerzo de firmes flexibles, cuya justificación cuanti. tativa será objeto de un próximo artículo.

$514 \cdot 54$
\end{abstract}

(Continuación)

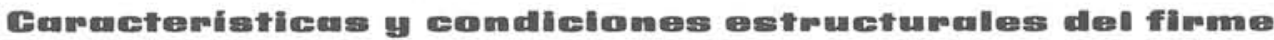

Hay que distinguir entre las caracteristicas estructurales del firme, que son parámetros representativos de su resistencia estructural, invariables en el tiempo mientras no se proceda a un acondicionamiento superficial o a un refuerzo, y las condiciones estructurales, que son características variables con las circunstancias ecológicas; las primeras son primordialmente de naturaleza descriptiva (espesores y materiales constitutivos de las distintas capas).

\subsection{COMPOSICION DE UN FIRME FLEXIBLE NUEVO}

Los firmes flexibles se componen de capas delgadas de diversos materiales, cuya calidad generalmente decrece con la profundidad hasta llegar a la explanada (terreno natural o artificial). A efectos de todo lo que sigue, se puede admitir la siguiente clasificación de las capas de un firme nuevo:

A) Pavimento: conjunto de capas superficiales de naturaleza homogénea, sobre la que se efectúa la rodadura de los vehículos. En él se pretenden: impermeabilidad, resistencia al desgaste, rozamiento elevado, y regularidad de rodadura. Actualmente pueden distinguirse tres tipos de pavimento:

- inexistente: la rodadura se efectúa entonces sobre la base. Sólo para tráfico ligero;

- tratamientos superficiales: bituminosos por lo general. Su reducido espesor hace que su valor estructural pueda considerarse nulo. Para tráfico medio;

- mezclas bituminosas: de distintas texturas, generalmente se organizan en capas de reducido espesor, denominándose "de rodadura» a la superior e «intermedias" a las demás. Puede existir, asimismo, una "capa de regularización» destinada a absorber las irregularidades de la base. Se utiliza para tráfico pesado.

B) Firme propiamente dicho: conjunto de capas cuya misión es repartir sobre la explanada las cargas que el tráfico transmite al pavimento, de manera que las solicitaciones a que se ven sometidos el firme y la explanada no sean perjudiciales. Existen varios tipos de afirmado, que se pueden combinar entre sí respetando la regla de la calidad decreciente con la profundidad. Ordenados con arreglo a dicha regla, se pueden distinguir los siguientes tipos:

- bases bituminosas: constituidas por mezclas bituminosas, análogas a las de los pavimentos, o por mezclas «in situ», entre las que se incluyen los tratamientos por penetración, etc., cuya calidad es inferior;

- bases estabilizadas con cemento: cuyo material básico puede ser una grava (grava-cemento) o un suelo (suelo-cemento);

- bases granulares de áridos machacados: con granulometría continua (zahorra artificial) o discontinua (macadam). Las primeras pueden estar estabilizadas con cloruros, lo cual no afecta a sus características estructurales, pero sí a la permanencia de éstas; 
- sub-bases estabilizadas: procedentes de áridos naturales o suelos, y cuyo estabilizante puede ser variado: cemento, ligantes bituminosos, cal, cloruros, etc.;

- sub-bases granulares: constituidas por áridos naturales;

- explanadas mejoradas: constituidas por suelos seleccionados.

C) Explanada: se considera homogénea e indefinida, al menos en la profundidad afectada por la transmisión de las cargas del pavimento.

\subsection{COMPOSICION DE UN REFUERZO}

Un refuerzo que se realice sobre un firme existente puede, según su importancia, asumir el aspecto de una repavimentación o el de un reafirmado. En el primer caso es imprescindible el empleo de mezclas bituminosas, puesto que los tratamientos superficiales no poseen valor estructural; en el segundo pueden utilizarse teóricamente todas las clases de firmes enumeradas en el apartado anterior; pero en la práctica, consideraciones mecánicas (el respeto de la regla de la calidad decreciente con la profundidad), económicas (relacionadas también con la existencia de espesores mínimos constructivos) o de facilidad de construcción (especialmente interesantes cuando el tráfico sea intenso y no se pueda desviar fácilmente) circunscriben el campo de elección a unos pocos tipos.

\subsection{CARACTERISTICAS ESTRUCTURALES DE LAS DISTINTAS CAPAS}

Un firme flexible, incluso ya reforzado, se reduce, estructuralmente hablando, a un sistema multicapa sometido a cargas dinámicas. Por lo tanto, para el conocimiento del plexo de tensiones, deformaciones y corrimientos producidos por dichas cargas, es teóricamente necesario el conocimiento de las características reológicas de cada capa, de las condiciones de deslizamiento entre capas, y de los estados límite de tensiones o deformaciones.

\subsubsection{Características reológicas de las capas de un firme flexible}

Existe una relación, característica de cada material, entre las tensiones aplicadas al mismo y las deformaciones en él producidas: la deformabilidad. Se puede distinguir entre la deformabilidad longitudinal, en la que se consideran las deformaciones paralelas a la línea de acción de la tensión aplicada, y la deformabilidad transversal, en que se consideran las deformaciones perpendiculares a dicha línea de acción.

\subsubsection{Deformabilidad longitudinal}

Las deformaciones producidas por la aplicación de una tensión pueden clasificarse en recuperables y remanentes, según que desaparezcan o no una vez cese de actuar la tensión, y en instantáneas y diferidas, según se produzcan simultáneamente a la aplicación de la tensión, o transcurra un cierto tiempo hasta que alcancen su valor definitivo.

Un material que sólo presenta deformaciones recuperables instantáneas se denomina elástico; un material que presente sólo deformaciones remanentes se denomina plástico; y un material que sólo presente deformaciones diferidas se llama viscoso. Estos materiales teóricos no existen, y en la realidad los materiales tienen comportamientos que participan de los tres tipos (visco-elasto-plásticos), según modelos reológicos más o menos complejos.

Las deformaciones remanentes no son deseables, puesto que, al no ser uniformes en todo el ancho de la carretera ni las solicitaciones del tráfico ni las características estructurales del firme, dichas deformaciones remanentes serán forzosamente diferenciales, y la regularidad transversal de la carretera se vería afectada (rodadas). Por lo tanto, se procura que la mayor parte de las deformaciones remanentes instantáneas tengan lugar durante la construcción del firme, sometiendo a éste a un proceso mecánico (compactación); y en cuanto a las deformaciones remanentes diferidas, se procura reducirlas haciendo que la viscosidad de los materiales empleados sea grande en relación con la velocidad de aplicación de las cargas del tráfico.

La deformabilidad se dice lineal si existe proporcionalidad entre las tensiones aplicadas y las respectivas deformaciones producidas, resultando así admisible la superposición de efectos cuando se superponen las causas.

En los materiales empleados en firmes flexibles se puede admitir que, al menos dentro del dominio de 
tensiones admisibles, la deformabilidad es lineal. Siempre dentro de dicho dominio, se puede esquematizar el comportamiento reológico de los distintos materiales bajo la acción de las cargas del tráfico con arreglo a lo que sigue:

a) los suelos de la explanada, y los materiales granulares, presentan una deformabilidad instantánea que consta de dos fases: elástica y plástica. La primera se define por el módulo de Young E (fig. 14).

La deformación plástica, cuya mayor parte tuvo lugar durante la construcción (compactación), puede definirse por un módulo análogo. Resulta interesante observar que, puesto que la deformación plástica tiene como consecuencia una mejor estructuración del material granular, que además se ve limitada físicamente por la granulometría, su cuantía va disminuyendo progresivamente al aumentar el número de ciclos de carga; y paralelamente va aumentando el módulo de Young.

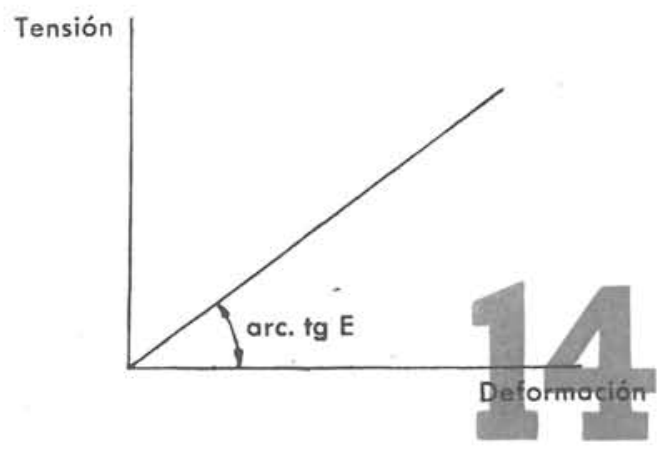

En los suelos, además, la humedad (grado de saturación) tiene una gran influencia en la deformabilidad, puesto que el agua actúa como lubricante o aglutinante entre las partículas.

b) los materiales hidráulicamente conglomerados presentan una deformabilidad cuya mayor parte es elástica, aunque pueden tener lugar hasta un cierto límite deformaciones viscosas remanentes muy diferidas (fluencia). El módulo de Young, representativo de la fase elástica, suele aumentar con la edad del material y. con la dosificación de conglomerante;

c) los materiales aglomerados con ligantes bituminosos tienen una deformabilidad compleja, en la que coexisten deformaciones elásticas y viscosas; estas últimas, tanto recuperables como remanentes. La definición de esta deformabilidad se basa en las curvas de fluencia (deformación a lo largo del tiempo, con tensión constante) y de relajación (tensión a lo largo del tiempo, con deformación constante) (fig. 15).

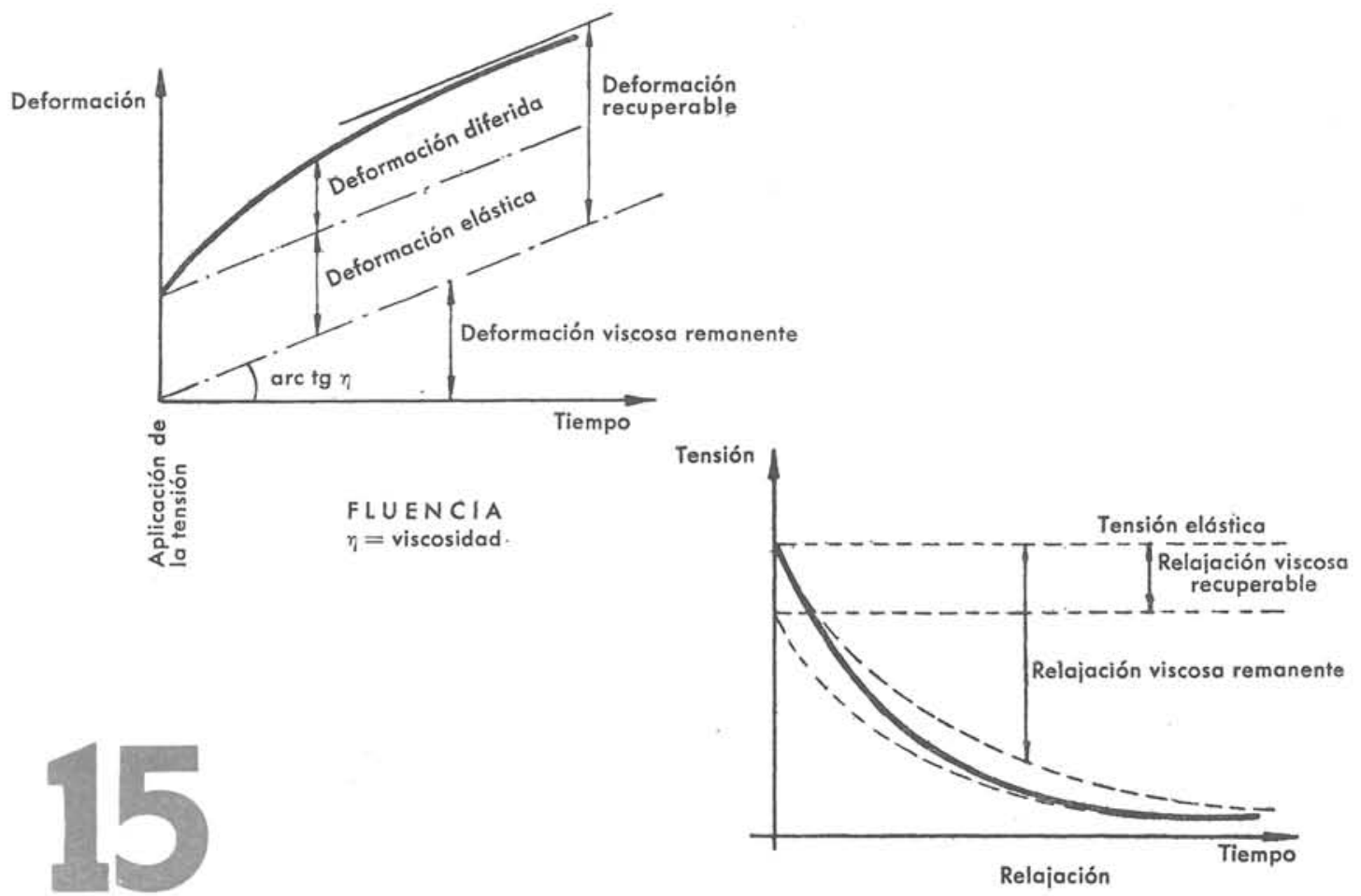


A partir de estas curvas pueden deducirse, para un determinado tipo de mezcla bituminosa:

- el módulo de Young, correspondiente a la fase elástica;

- el coeficiente newtoniano de viscosidad, correspondiente a la fase viscosa remanente;

- la ley de la deformabilidad viscosa recuperable.

Estas características se modifican sustancialmente con la temperatura del material, y secundariamente por el envejecimiento del ligante bituminoso.

Resumiendo: la deformabilidad longitudinal de un material frente a la aplicación de una tensión puede representarse siempre por un módulo de deformabilidad dinámico complejo, cuya magnitud es función de la velocidad de aplicación de la tensión, y del estado del material (historial previo de cargas, temperatura, etc.).

\subsubsection{Deformabilidad transversal}

Independientemente de su naturaleza (recuperable o remanente, instantánea o diferida), a cada deformación longitudinal corresponde una deformación transversal, perpendicular a la dirección de la aplicación de la tensión; la cuantía de esta deformación transversal depende del coeficiente de Poisson.

\subsubsection{Condiciones de deslizamiento entre capas}

Debido a la irregularidad de las superficies de las capas, resultante de la construcción de las mismas, se suele admitir que no se pueden producir deslizamientos entre ellas. Unicamente puede considerarse tal posibilidad:

- cuando la diferencia de deformabilidades entre las dos capas adyacentes sea muy grande;

- para tiempos de carga muy largos;

- cuando en la superficie de separación haya una cantidad grande de un material deslizante (p. ej., un riesgo de imprimación o adherencia).

Las condiciones de resistencia son peores cuando se producen deslizamientos, por lo que se tiende a evitar las circunstancias anteriores.

\subsubsection{Estados límite}

Para cada material existen unos límites de tensión y/o deformación, rebasados los cuales su deformabilidad deja de ser lineal; esto coincide con la aparición de deformaciones remanentes importantes, y consecuentemente con la ruina estructural del firme a más o menos corto plazo. Por consiguiente, el dimensionamiento del firme, como sistema estructural multicapa, debe realizarse de manera que en ninguna de las capas se alcancen dichos estados límite bajo la acción de las cargas del tráfico.

Las deformaciones remanentes suelen concentrarse, en los materiales conglomerados, en forma de fisuras que modifican la definición geométrica de la capa y, por tanto, su plexo de tensiones-deformaciones-corrimientos bajo carga.

En la definición de los estados límite tiene intervención el concepto de fatiga, que, a su vez, está relacionado con la acumulación de unas pequeñas deformaciones remanentes en cada ciclo de carga: recuérdese que en los materiales granulares se ha mencionado ya este fenómeno; y en los materiales conglomerados y aglomerados, aunque la viscosidad es grande en relación con la velocidad de aplicación de las cargas, se produce también una pequeña deformación remanente. Resulta cómodo, por lo tanto, simplificar el problema trasladando el tener en cuenta las deformaciones remanentes a la definición de "estado límite» en función del número de ciclos de carga, y estudiando la deformabilidad como puramente recuperable.

\subsection{ANALISIS ESTRUCTURAL DEL FIRME FLEXIBLE}

Las cargas del tráfico aplicadas sobre la superficie del pavimento, bajo forma de una presión sobre un área que se desplaza, inducen en el sistema multicapa un plexo de tensiones, deformaciones y corrimientos, que es función:

- de las características reológicas de las distintas capas;

- de la velocidad, forma y presión del área cargada. 
Dicho plexo ha de estar contenido dentro de los límites admisibles, si es que el firme está correctamente

dimensionado.

Las características reológicas de las distintas capas han sido ya estudiadas en el apartado 6.3 ; en cuanto a las del área cargadas por el tráfico, se suele admitir que la forma de la huella de un neumático es circular y su presión uniforme, aunque esto no corresponda exactamente a la realidad.

El aparato matemático desplegado para la determinación del plexo de tensiones-deformaciones-corrimientos es notablemente complicado (por el gran número de parámetros que en él intervienen), aun en el caso simple de carga estática, en el que no entran en juego las deformaciones diferidas. La resolución teórica del caso dinámico no ha podido ser lograda todavía.

Si se añade a este fracaso matemático la incertidumbre en la definición (experimental) de las características reológicas de las distintas capas, se puede comprender el favor que gozan las caracterizaciones empíricas del plexo tensio-deformacional, que además presentan la ventaja de una mayor sencillez formal.

\subsection{DETERMINACIONES DE LAS CARACTERISTICAS REOLOGICAS DE LOS MATERIALES}

La determinación de las características reológicas de las distintas capas debe tener una base forzosamente experimental, aunque esta experiencia luego pueda condensarse en fórmulas que presenten una estimación «a priori» a partir de ciertos datos. La experimentación puede realizarse sobre probetas de laboratorio, o en el propio firme (auscultación). La determinación de los estados límite exige generalmente ensayos destructivos.

En el primer caso, la diversidad geométrica entre la probeta y la capa del firme, y entre la técnica operatoria y la solicitación real, puede, en ocasiones, poner en duda la representatividad del experimento. En el caso de una auscultación, ésta puede resultar extremadamente incómoda, puesto que hay que instalar a pie de obra todo un laboratorio móvil. En ambos casos, además, se plantea el problema de la representatividad que un reducido número de ensayos puede tener de las propiedades de un material forzosamente muy extenso y variable.

De estas consideraciones surge claramente la necesidad de que los ensayos que sirven de base para caracterizar las propiedades reológicas de las capas del firme sean de fácil y rápida ejecución, aunque su representatividad se base en correlaciones estadísticas con dichas propiedades, con objeto de poder disponer de una información más completa sobre la varìabilidad de los materiales.

En laboratorio, la determinación de los estados límite se efectúa por rotura de probetas bajo condiciones normalizadas, y la determinación de la deformabilidad se realiza o bien directamente o bien por estudio de las características de transmisión de vibraciones.

Las auscultaciones sobre firmes (prescindiendo de la obtención de probetas que luego se ensayan en laboratorio) caracterizan la deformabilidad por el estudio de la transmisión de vibraciones, o por los corrimientos superficiales bajo carga (ensayos de placa y deformaciones puntuales); la determinación de estados límite requiere la prosecución de los ensayos hasta un límite destructivo, en tramos experimentales. Hay que observar, además, que las auscultaciones no pueden referirse a una capa aislada, sino a un conjunto de capas, y que luego hay eventualmente que desglosar la influencia de una u otra capa en el resultado final.

\subsection{AUSCULTACION DE LAS DEFORMACIONES PUNTUALES}

Por su facilidad de realización, que permite la obtención de una información muy representativa de las diversas zonas del firme, y suficientemente numerosa como para aplicar la Estadística para interpretar sus resultados, han obtenido un gran éxito en los últimos años las auscultaciones realizadas midiendo el corrimiento vertical de la superficie del pavimento bajo un eje real de vehículo pesado. Este ensayo se denomina generalmente de «deformación puntual» o de «deflexión» (anglicismo por el anterior).

Aunque nada se opone teóricamente a que el ensayo sea dinámico, la facilidad de realización obliga a que resulte un ensayo estático, es decir, desarrollado a lenta velocidad, lo que hace intervenir las deformaciones diferidas a corto plazo. Existen varias modalidades, de las que unas estudian la rama de carga y otras la de descarga (recuperación), siendo estas últimas las que mejor se correlacionan con el comportamiento del firme; confirmando la hipótesis de que dicho comportamiento depende de las facultades de «recuperación" del firme tras la solicitación de la carga. 
En la deformación puntual influyen principalmente:

- el tipo y la carga de eje empleado: se suelen normalizar éstos para el ensayo, aunque, en la realidad, los ejes que transitan tienen tipos y cargas muy distintos;

- las características estructurales de las capas del firme: siendo su espesor constante, son las características reológicas las que intervienen, y principalmente son dos las causas de variación: la temperatura, que modifica la rigidez de las mezclas bituminosas, y la humedad, que modifica la deformabilidad de la explanada.

\section{Comportamiento del firme flexiblo}

\subsection{COMPORTAMIENTO DEL FIRME NUEVO}

Un firme nuevo, con un índice de servicio elevado, es abierto al tráfico; y como consecuencia del paso de cada uno de los ejes que integran el tráfico pesado se va produciendo una disminución de dicho índice de servicio (v. apartado 4.2), cuya magnitud depende de las condiciones estructurales del firme en el momento del paso del eje. Como ya se ha visto (v. 6.6) que la deformación puntual (dinámica) causada por el eje es representativa de las condiciones estructurales del firme, se puede admitir que la disminución del índice de servicio está asociada de alguna forma a las deformaciones puntuales impuestas por el tráfico.

Por lo tanto, el índice de servicio irá disminuyendo, con velocidad variable, según evolucione la frecuencia del tráfico (aproximadamente constante) y las condiciones ecológicas (fundamentalmente variables), hasta alcanzar el tope mínimo admisible y aun rebasarlo, si no se emprende una acción remediadora, hasta poner el firme fuera de servicio.

\subsection{VALOR ESTRUCTURAL REMANENTE}

En un momento cualquiera del proceso descrito en el apartado anterior, se puede definir el valor estructural remanente del firme como igual a las características estructurales de un firme nuevo que, si se abriera al tráfico en ese momento, llegaría a ponerse fuera de servicio, por la acción del mismo tráfico, simultáneamente al primer firme. Naturalmente, el valor remanente, sea cual fuere el índice empleado para caracterizarlo, es inferior a las características estructurales originales del firme, y coincide con el que tendría un acondicionamiento superficial, considerado como firme nuevo (fig. 16).

\subsection{COMPORTAMIENTO DEL REFUERZO}

La adición de un refuerzo en un instante determinado de la vida útil de un firme viene a sumar sus características estructurales con el valor estructural residual y, por consiguiente, prolonga el período durante el cual el firme puede mantenerse en servicio. Si el refuerzo se efectúa, como debe ser, antes de que el firme alcance el tope mínimo admisible, también se prolongará el tiempo ("período de proyecto») necesario para que se alcance dicho tope (fig. 17).

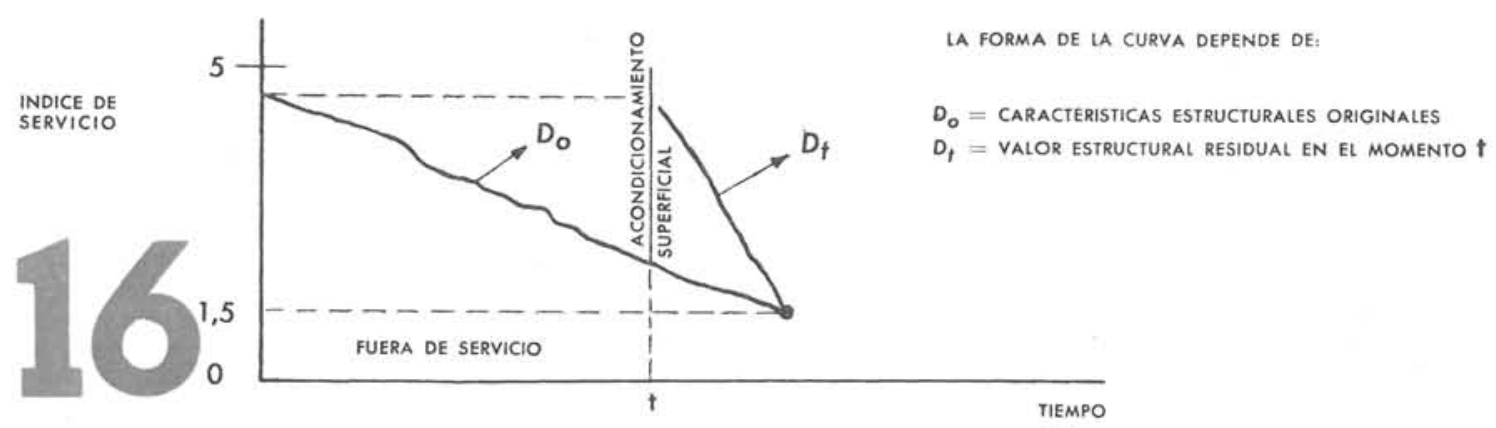


La disminución del índice de servicio, o sea, el deterioro del pavimento, antes de que llegue a ser tan grave que imponga un refuerzo o una reconstrucción, puede ir siendo paliada por operaciones de conservación, consistentes en mejoras localizadas, generalmente no estructurales (del tipo de los acondicionamientos superficiales). Los costes de conservación dependen de la política que se fije para ésta.

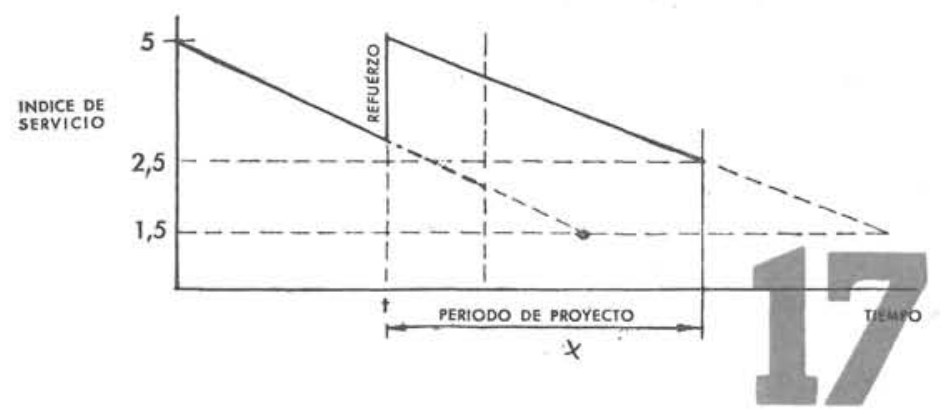

Actualmente en España se fijan «a priori» las asignaciones medias de conservación por kilómetro en función de la IMD total, reconociendo así implícitamente que a un mayor tráfico corresponde un mayor desgaste. Hay que observar que en dichas asignaciones están incluidos conceptos no relacionados con el deterioro del pavimento, como son las plantaciones y la señalización. En la realidad, estas asignaciones medias se deberían multiplicar por un coeficiente de asignación que es función del estado de la carretera (englobando aquí de nuevo conceptos extraños al firme propiamente dicho).

Un enfoque racional del problema podría efectuarse según el esquema siguiente: debido a la falta de uniformidad del firme (especialmente de la explanada), la disminución del índice de servicio de un tramo no es uniforme en todo él, sino que ciertas zonas van alcanzando antes el tope mínimo admisible (v. apartado 9.2). La política correcta de conservación consiste en acondicionar superficialmente estas zonas a medida que se van produciendo, devolviéndoles un índice de servicio elevado, aunque sin aumentar su capacidad estructural, hasta que el índice de servicio de la totalidad del tramo alcance el tope mínimo, en cuyo momento se refuerza todo el tramo (fig. 18).

La contabilización de estas operaciones de conservación da origen a una curva de «gastos de conservación acumulados» en función del «índice de servicio global» y de la heterogeneidad del firme.

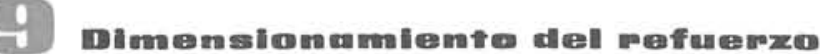

\subsection{CASO IDEAL: TRAMO UNIFORME}

Supóngase que el tramo de carretera que se desea reforzar es lo suficientemente corto como para que sus características y condiciones estructurales puedan considerarse uniformes, es decir, únicamente variables en el tiempo y no en el espacio.

A un dimensionamiento dado del refuerzo corresponderán:

- un coste de construcción;

- una curva de disminución de evolución del índice de servicio con el tiempo que, a su vez, tendrá como consecuencias:

- un coste de conservación creciente,

- un coste de explotación del transporte (ligero y pesado), también creciente.

Un dimensionamiento correcto debería hacer que la suma de estos costes, actualizada al mismo instante, fuera mínima. También habría que tener en cuenta algunas limitaciones de otra índole, por ejemplo que el período de proyecto resultante no fuera demasiado corto, o que el dimensionamiento resultante no fuera tan grande que hiciera más aconsejable una reconstrucción. Estas limitaciones se describen más adelante. 


\subsection{CASO REAL SIMPLIFICADO: TRAMO HOMOGENEO}

En la realidad, uno de los datos de partida, la capacidad estructural de un firme antiguo homogéneo (representado, por ejemplo, por su deformación puntual máxima), no es conocido más que a través de un muestreo que determina unos cuantos valores puntuales y dispersos, siendo de presumir la existencia de valores tanto superiores como inferiores a la media de los obtenidos. Comoquiera que las características estructurales del refuerzo no pueden variarse en el espacio con una flexibilidad que permitiera en cada punto disponer el refuerzo estrictamente necesario (aun admitiendo que el resultado obtenido en cada punto es auténticamente representativo de las condiciones estructurales del firme antiguo en dicho punto), es inevitable la existencia de zonas supra- e infradimensionadas para un refuerzo dado. La economía exige que, tanto unas como otras, estén contenidas dentro de ciertos límites admisibles đe proporción.

Aplicando las teorías de la Estadística, y en el supuesto de que las medidas obtenidas de la capacidad estructural del firme antiguo sean muestras de una sola población (o sea, de un conjunto que responda a una ley de distribución única), se puede hallar, con una probabilidad de acierto dada y para un refuerzo determinado, la proporción de firme insuficientemente reforzado (fig. 19).

La probabilidad de acierto aumenta si se dispone de una mejor información, por ejemplo si el número de medidas de la muestra aumenta, o si se conoce «a priori» la ley de distribución de la población.
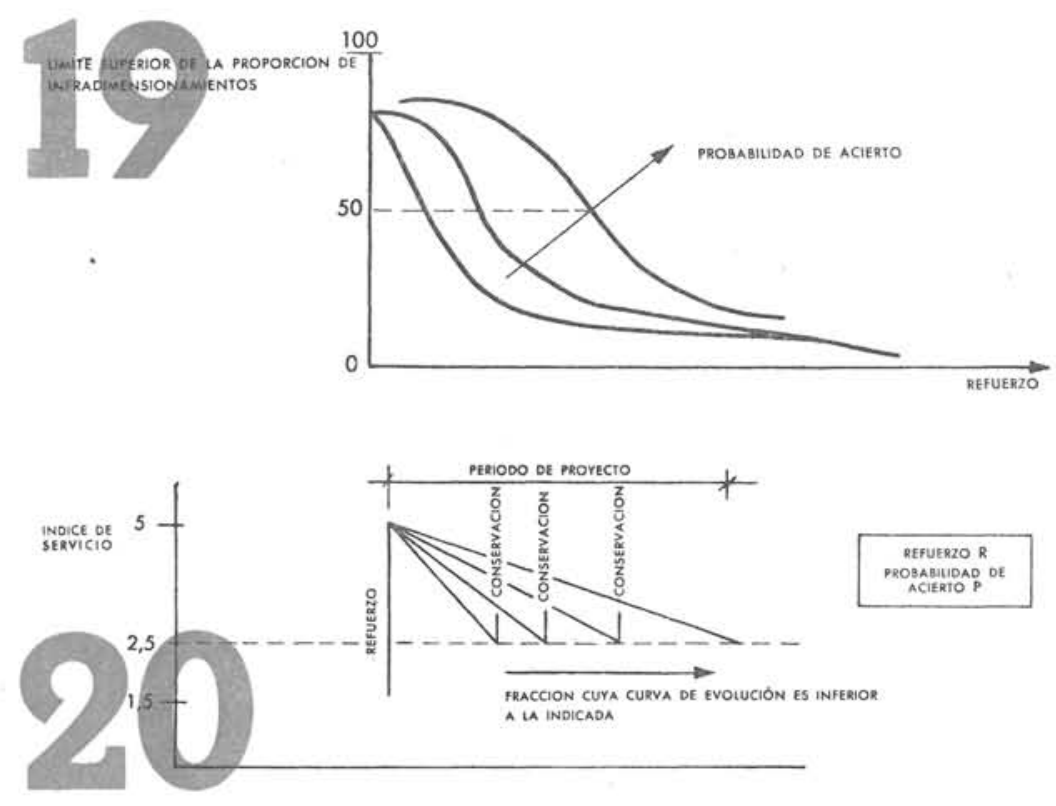

En relación con esta última, se comprueba que la exactitud de la hipótesis de distribución que se formule no tiene una gran repercusión en los resultados del estudio.

Considerando el tramo homogéneo (una sola población) como un conjunto de tramos puntuales uniformes, se puede, para un dimensionamiento dado del refuerzo, establecer una familia de curvas de disminución del índice de servicio que corresponderá, con una probabilidad de acierto dada, a las diversas fracciones del tramo (fig. 20).

Antes de que transcurra el "período de proyecto" del refuerzo, todas las zonas que alcancen el tope mínimo del índice de servicio se reacondicionarán superficialmente mediante conservación.

A partir de esta familia de curvas de evolución del índice de servicio, se pueden hallar los costes de explotación y de conservación globales del tramo homogéneo (por integración de los costes uniformes correspondientes a cada fracción del tramo), y minimizar el coste total (incluido el de construcción); obteniendo así el refuerzo óptimo del tramo, con una determinada probabilidad de acierto (o sea, de que el coste no sea superior al previsto). Las variables independientes en dicha optimización son el dimensionamiento del refuerzo y el período de proyecto considerado, y la variable dependiente es el costo total actualizado (construcción + conservación + explotación) a lo largo del período de proyecto.

\subsection{CASO REAL: TRAMO HETEROGENEO}

La dificultad de aplicar el esquema descrito en el apartado anterior estriba en que en un mismo tramo coexistan varias «poblaciones», correspondientes a distintas causas de variabilidad en el firme antiguo. La separación en subtramos consecutivos es difícil, y debe basarse en criterios estadísticos, de manera que se identifique con la máxima perfección posible cuáles son los subtramos realmente distintos, y que cada uno de ellos tenga una probabilidad elevada de proceder de una sola población, del tipo que se haya determinado. Por otro lado, la longitud de estos subtramos, a efectos de la uniformidad del refuerzo, es deseable sea la mayor posible; aunque no es de descartar la posibilidad de aplicar remedios localizados (por ejemplo, saneamiento de un blandón) a subtramos muy cortos y en muy mal estado. 


\subsection{ESPESOR EQUIVALENTE}

En el capítulo anterior se ha tratado del dimensionamiento del refuerzo, sin distinguir el hecho de que éste puede estar compuesto por distintos espesores de diversos materiales. Cuando se trata de dimensionar "a priori», con objeto de reducir el número de variables a considerar, es corriente utilizar el concepto del «espesor equivalente».

Según este concepto, un determinado espesor de un material puede ser sustituido por un espesor equivalente de otro material que presente la misma "capacidad estructural». Esto representa una simplificación, puesto que en la realidad la capacidad estructural depende también de las rigideces relativas y de la disposición de las demás capas del firme; pero es muy cómodo, porque el dimensionamiento del refuerzo se condensa en un solo parámetro: el espesor equivalente de un material-patrón (se suele elegir la mezcla bituminosa cerrada debido a la frecuencia de su empleo).

El dimensionamiento del refuerzo se distribuye entre los distintos materiales, en función de sus coeficientes de equivalencia. Deben respetarse ciertas reglas de distribución, si no se quiere alterar peligrosamente la validez de la simplificación introducida, o tropezar con dificultades funcionales o constructivas.

\subsection{LIMITACIONES EN LA ELECCION DE MATERIALES}

\subsubsection{Espesores}

Al ser de naturaleza granular los materiales empleados para la construcción de refuerzos, el espesor de las tongadas constructivas deberá estar relacionado con el tamaño máximo de sus partículas, de manera que las mayores no impidan la compactación de las demás (límite inferior) ni, en el caso de mezclas bituminosas (fluidificadas por el ligante) se pierda estabilidad al compactar (límite superior).

Además existen mínimos de espesor por razones constructivas $(10 \mathrm{~cm}$ en materiales puramente granulares, $15 \mathrm{~cm}$ en conglomerados y $3 \mathrm{~cm}$ en mezclas bituminosas) o de otra índole (por ejemplo: para evitar la transmisión de las fisuras de contracción de las capas hidráulicamente conglomeradas inmediatamente subyacentes, el espesor de mezclas bituminosas debe ser superior a un cierto límite).

\subsubsection{Calidades}

Respondiendo a la disminución de las solicitaciones con el aumento de la distancia a la superficie de aplicación de las cargas, es admisible, y económicamente interesante, el utilizar materiales de menos calidad estructural (coeficiente de equivalencia), a medida que se trate de capas más profundas, hasta alcanzar la explanada (firme nuevo) o el firme antiguo (refuerzo).

En este último caso hay que tener en cuenta el efecto de «yunque» que pueda causar la presencia de un firme antiguo cuya calidad sea mayor que la de la capa inferior del refuerzo.

Hay que tener muy presente que la transición de calidad no puede ser nunca brusca; porque si lo fuera, la capa superior (de material de mayor calidad) se verá sometida a importantes solicitaciones que pudieran llegar a ser inadmisibles. Por ello, se suele exigir que la relación de rigideces entre dos capas consecutivas no rebase un cierto límite.

\subsubsection{Pavimento}

La necesidad funcional de la rodadura impone la presencia de un pavimento que, salvo el caso de tráfico muy ligero (que rara vez dará origen a un refuerzo), limita la elección a un tratamiento superficial o una mezcla bituminosa. Sin embargo, el tratamiento superficial tiene también una limitación de empleo en función del tráfico pesado, por lo que por encima de un cierto nivel de tráfico sólo se pueden emplear mezclas bituminosas de alta calidad, en espesores tanto mayores cuanto más intenso y pesado sea el tráfico.

\subsubsection{Limitaciones constructivas}

Las características propias del proceso de ejecución de las distintas clases de refuerzo pueden influir en su elección. Una de estas características es la rapidez de ejecución, cuya importancia en presencia de un tráfico, a veces indesviable, es primordial; otra característica se refiere a las limitaciones de tipo climatológico, que impiden trabajar durante ciertas épocas del año; una tercera es la de que, por encima de un determinado espesor de refuerzo puede ser más conveniente no reforzar el firme, sino reconstruirlo. 


\section{Bases d'une méthode rationnelle pour le dimensionnement des renforce- ments de revêtements souples, basée sur la mesure de la déflexion sous axe chargé}

Sandro Rocci, ingénieur des Ponts et Chaussées

Cet article est une description qualitative des facteurs intervenant dans le comportement du revêtement routier et des rapports existant entre eux. Un schéma fonctionnel en est établi, dont l'objectif ultime économique est la réduction au minimum du coût total (exécution + entretien + exploitation) d'une route. Sont également établies les bases pour la création d'une méthode de dimensionnement des renforcements pour les revêtements souples, dont la justification quantitative sera l'objet d'un prochain article.

\section{Basic data to define a national system of designing overlays of flexible pavements, based on deflection under a loaded axle}

Sandro Rocci, civil engineer

This article gives a qualitative description of the factors that influence in the behaviour of a road pavement, and of the relations between these factors. A functional pattern is established, whose final economic aim is the reduction of the overall cost (construction plus maintenance plus exploitation) of a road. Further, principles are established for the design of overlays for flexible pavements: the quantitative justification of these criteria will be discussed in another article.

\section{Grundlagen einer rationalen Methode für die Dimensionierung von Beans- pruchungen von schmiegsamen Decken, die auf der Messung der Punktver- formung unter einer belasteten Achse gegründet wird}

Sandro Rocel, Bauingenieur

Dieser Artikel stellt eine qualitative Beschreibung der Faktoren, die in der Verhaltung der Strassendecken vorkommen, und der Beziehungen unter ihnen dar. Man setzt ein Schema fest, dessen letzten economischen Zweck die minimalen Gesamtkosten einer Strasse (Bau, Aufbewahrung und Betrieb) ist. Gleichzeitig setzt man die Grunlagen für eine Dimensionierungsmethode von Beanspruchung von schmiegsamen Decken fest, die auf der Messung der Punktverformung unter einer belasteten Achse gegründet wird; der quantitative Nachweis davon wird das Thema für den nächsten Artikel sein. 